\title{
Antisaccade costs with static and dynamic targets
}

\author{
RichaRd GODIJN AND ARTHUR F. KRAMER \\ University of Illinois at Urbana-Champaign, Urbana, Illinois
}

\begin{abstract}
In the present study we examined the antisaccade cost (latency difference between antisaccades and prosaccades) in a variety of search tasks. In a series of experiments participants searched for a target and were required to execute a saccade toward (prosaccade) or away (antisaccade) from the target. The results revealed that the antisaccade cost was greater for static targets than for dynamic targets, and it was greater for onset targets than for offset targets. Furthermore, the offset of an onset target interfered with prosaccades, but facilitated antisaccades, resulting in a reduction of the antisaccade cost. To account for the data a model is presented, in which attentional control and working memory processes play an important role in the generation of antisaccades.
\end{abstract}

When observers visually examine their environment they direct their attention toward objects that are of interest. Although attention can be directed to a region of the visual scene covertly without moving the eyes (e.g., Posner, 1980), observers routinely shift their attention overtly by moving their eyes to regions of interest several times a second. Despite the strong and natural link between attention and eye movements there is overwhelming evidence that observers can attend to an object and subsequently direct their gaze to a different location without first moving their eyes to the attended object.

In a classic study Hallett (1978) developed the socalled antisaccade task, in which participants are required to move their eyes away from a peripheral onset. In the original version of this task participants fixate an object in the center of the screen, which abruptly steps to the left or the right. In the antisaccade condition the task is to move the eyes in the opposite direction of the step. For example, if the object moves to the left the participant is required to move their eyes to the right. In another version of this antisaccade task (typically referred to as the overlap condition) there is no movement of a fixation object, but a peripheral onset appears while the central fixation point remains on the screen. Hallett (1978) observed that after a certain degree of practice participants were quite good at executing antisaccades. However, on some trials participants execute erroneous saccades (prosaccades) toward the onset, despite the instruction to move their eyes in the opposite direction. Subsequent research has shown that the percentage of erroneous prosaccades varies widely depending on the specific characteristics of the task (Fischer \& Weber, 1992, 1996; Everling \& Fischer, 1998; Mokler $\&$ Fischer, 1999).

Performance of participants in the antisaccade task is often compared with performance in the prosaccade task, in which the task is to execute a saccade toward the peripheral onset. It is consistently found that there are fewer sac- cade errors in the prosaccade task than in the antisaccade task and correct prosaccade latencies are typically shorter than correct antisaccade latencies. The latency difference between prosaccades and antisaccades (the antisaccade cost) suggests that different processes may be involved in these two types of saccades.

It is typically assumed that when an onset appears in the visual periphery the prepotent response is to move the eyes toward the onset. In fact, the prosaccade is often described as being executed reflexively. When an antisaccade is required the reflexive prosaccade must be inhibited and a saccade must be programmed voluntarily (or endogenously) in the opposite direction. Therefore, it has been argued that antisaccades are slower than prosaccades because they are programmed voluntarily instead of reflexively (e.g., Walker, Walker, Husain, \& Kennard, 2000; Massen, 2004), or because of the extra time needed to inhibit a prosaccade (e.g., Olk \& Kingstone, 2003; Pratt $\&$ Trottier, 2005).

According to one view of the latency difference between antisaccades and prosaccades (the antisaccade cost) two saccades are programmed in parallel in the antisaccade task, a reflexive prosaccade and a voluntary antisaccade (e.g., Mokler \& Fischer, 1999; Massen, 2004). The programming of the antisaccade is initiated as soon as the location of the stimulus is known. It is assumed that the programming of the antisaccade occurs without a delay caused by the time needed to inhibit the prosaccade. In a recent study, Massen (2004) tested the idea that reflexive prosaccades and voluntary antisaccades are programmed in parallel in the antisaccade test. Massen reasoned that if prosaccades and antisaccades are programmed independently and in parallel, the error rate in the antisaccade task depends on the relative processing times of these two programs. For example, if the antisaccade is slowed down the error rate should increase, while if the prosaccade is slowed down the error rate should decrease. In Experiment 1 of her study,

R. Godijn, godijn@uiuc.edu 
Massen provided evidence for this idea by manipulating the relative frequency of prosaccades and antisaccades. Based on the orientation of a central cue participants were required to execute a prosaccade or antisaccade, while the relative frequency of antisaccades was varied between blocks. The results showed that, while prosaccade latencies were unaffected by the probability manipulation, there was a negative relationship between antisaccade latency and antisaccade frequency. Thus, as antisaccade frequency decreased, the programming time of antisaccades increased while the programming time of prosaccades remained constant. Furthermore, slowing down the antisaccade resulted in an increase in the proportion of erroneous prosaccades in the antisaccade condition, which was interpreted as evidence for the parallel programming hypothesis.

An alternative view of the antisaccade cost is that antisaccade latencies are longer than prosaccade latencies because of the time-consuming operation of inhibiting the reflexive prosaccade. This view assumes that the processes involved in antisaccades are more or less sequential. Specifically, it is assumed that a prosaccade must be inhibited before an antisaccade can be programmed. Evidence for this view was provided by Olk and Kingstone (2003) using a task in which the inhibition was equated between prosaccades and antisaccades. Participants were required to determine the orientation of a peripheral arrow and, depending on the orientation, execute a prosaccade or antisaccade. It was assumed that participants would need to inhibit a prosaccade in the prosaccade condition as well as in the antisaccade condition until the orientation of the arrow could be determined. It was found that equating the inhibition between the prosaccade condition and the antisaccade condition resulted in a significantly reduced antisaccade cost, which was taken as evidence that prosaccade inhibition accounts for a large proportion of the antisaccade cost.

Although there is currently no consensus whether the processes involved in antisaccades are performed serially or in parallel there does appear to be a consensus concerning the reflexive programming of prosaccades. That is, it is widely accepted that the appearance of the peripheral onset in the antisaccade task (as well as in the prosaccade task) results in the reflexive programming of a prosaccade (e.g., Everling \& Fischer, 1998; Hallett, 1978; Massen, 2004; Mokler \& Fischer, 1999; Olk \& Kingstone, 2003; Pratt \& Trottier, 2005; Walker et al., 2000). The different views merely disagree on whether the programming of the antisaccade occurs simultaneously with the reflexive saccade programming or after the reflexive saccade program has been inhibited. Although it is undisputed that onsets have a strong reflexive or exogenous effect on attention (Yantis, 1996; Theeuwes, 1995) and saccades (e.g., Godijn \& Theeuwes, 2002; Kramer, Hahn, Irwin, \& Theeuwes, 1999; Theeuwes, Kramer, Hahn, \& Irwin, 1998), it is equally clear that the onset is part of the task-set of participants. That is, participants have the goal of locating the onset in order to determine the correct antisaccade location. Therefore, it is possible that there is an endogenous component to prosaccades and that observers may on occasion execute erroneous prosaccades due to the taskrelevance of the onset.
We recently provided evidence for the idea that prosaccades are not completely reflexive using a task in which participants viewed displays containing both an onset and a color singleton presented together with two nononset distractors with the same color as the onset (Godijn \& Kramer, 2006). In separate blocks participants searched for the onset or color singleton and were required to execute a prosaccade or antisaccade in response to the search target. The results showed that the antisaccade cost was greater for color singletons than for onsets and there was a trend toward more prosaccade errors with color singleton search targets than with onset search targets. Furthermore, when the onset or color singleton was not the search target, but was instead presented as a distractor, it hardly ever elicited an erroneous saccade in its direction. These findings provided evidence that the task-relevance of the prosaccade stimulus can play a role in the production of erroneous prosaccades. In accordance with previous research we proposed that working memory plays an important role in the generation of antisaccades and the suppression of erroneous prosaccades. That is, when an onset or color singleton was task-relevant, and its identity was maintained in working memory, more erroneous saccades were executed in its direction. Evidence for a role of working memory in the antisaccade task comes from several lines of research.

First, Roberts, Hager, and Heron (1994) combined the antisaccade task with a concurrent working memory task and demonstrated that the percentage of erroneous prosaccades was increased in this dual-task situation. This provides evidence that when working memory is overloaded participants are often unable to execute the appropriate response.

Second, Kramer, Gonzalez de Sather, and Cassavaugh (2005; also see Munoz, Broughton, Goldring, \& Armstrong, 1998 ) found that the percentage of erroneous prosaccades decreased from age 8 to age 16. This pattern of results is consistent with the developmental time course of working memory, which continues well into the mid-teens. A third line of evidence comes from patient studies that have shown that patients with frontal lesions have an increased error rate in the antisaccade task (e.g., Guitton, Buchtel, \& Douglas, 1985; Milea et al., 2003; Pierrot-Deseilligny, Ploner, Muri, Gaymard, \& Rivaud-Pechoux, 2002; Pierrot-Deseilligny, Rivaud, Gaymard, \& Agid, 1991). Because these areas are involved in working memory (e.g., Asaad, Rainer, \& Miller, 1998; Goldman-Rakic, 1987; Rao, Rainer, \& Miller, 1997) these findings support the involvement of working memory in preventing erroneous prosaccades. Fourth and finally, Unsworth, Schrock, and Engle (2004; also see Kane, Bleckley, Conway, \& Engle, 2001) showed that participants with a low working memory capacity executed more erroneous prosaccades in the antisaccade task and had longer antisaccade latencies than participants with a high working memory capacity. Furthermore, in their Experiment 2 prosaccade and antisaccade trials were mixed within blocks and the correct response was indicated at the start of each trial through the identity of a fixation stimulus. Unsworth et al. found that participants with a low working memory capacity executed more erroneous antisaccades on prosaccade trials (i.e., saccades away from the target when they were 
required to execute a saccade toward the target) than participants with high working memory capacity and participants executed more erroneous antisaccades when the previous trial was an antisaccade trial than when it was a prosaccade trial. Thus, the deficit of low working memory participants was not in their ability to inhibit a saccade to the prosaccade location, but rather in their ability to maintain task goals in working memory and to engage in task-appropriate behavior. Engle and colleagues (e.g., Engle, 2000; Engle, Kane, \& Tuholski, 1999; Kane, Poole, Tuholski, \& Engle, 2006; Kane et al., 2001) have argued that individual differences in working memory capacity (or working memory span) should have the greatest effect on performance in situations in which active maintenance of task goals is needed, particularly when encountering strong environmental distractors or internal interference. According to this view the limitation in working memory does not necessarily concern the number of items in working memory, but instead, the limitation concerns the extent to which participants can control the focus of attention under a myriad of different situations.

In the present study we will test a model of antisaccade generation, in which working memory and attentional control play an important role. First, consistent with previous studies we propose that correct antisaccade generation requires correct maintenance and application of task goals in working memory. Second, following the controlled attention view of working memory of Engle and colleagues (e.g., Engle, 2000; Engle et al., 1999; Kane et al., 2001; Kane et al., 2006) we propose that attentional demands of a task affect working memory in the sense that an increase in attentional demands impairs the ability of working memory to maintain and apply the relevant task goals. This view can account for the surprising finding of Godijn and Kramer (2006) that the antisaccade cost is greater for color singletons than for onsets; the greater attentional demands of searching for a color singleton interfere with the working memory processes related to the task-goal of executing a saccade away from the target.

Recently, Pratt and Trottier (2005) reported findings that seem in conflict with this proposal. In their study Pratt and Trottier required participants to execute prosaccades or antisaccades in response to onset or offset targets. The results showed that the antisaccade cost was greater for onset targets than for offset targets. According to the authors this difference was attributable to the faster programming of reflexive saccades toward onsets relative to offsets. At first glance this finding appears inconsistent with our proposal, because we assume that the antisaccade cost depends to a large extent on the attentional demands of the localization of the target. The fact that onsets elicit faster prosaccades than offsets makes it implausible that localization of onsets is more attention-demanding than localization of offsets. However, we suggest that offsets are a special case, because they allow a fast disengagement of attention from the prosaccade location. That is, we assume that in order to localize the target attention is first directed to the prosaccade location. In order to generate an antisaccade attention is then disengaged from the prosaccade location and shifted to the antisaccade location. Consequentially, the speed of disengagement from the prosaccade location affects the antisaccade cost. Evidence for a relatively fast attentional disengagement from offset locations comes from a number of studies that have examined attentional capture and inhibition-ofreturn (IOR) with onset and offset stimuli (e.g., Pratt \& Hirshhorn, 2003; Samuel \& Weiner, 2001). These studies have shown that IOR occurs earlier in time at the location of an offset than at the location of an onset. Since it is assumed that attention must first be disengaged from a location before IOR can be revealed at that location, the difference in time course of IOR between offsets and onsets supports the notion that disengagement of attention from the location of an offset is relatively fast.

The goal of the present study is twofold. First, a series of experiments was designed to examine the effect of attentional demands on the antisaccade cost. Specifically, the antisaccade cost of various static (targets defined as the unique, unchanged object) and dynamic (onsets and offsets) targets was examined. According to the idea that the antisaccade cost is increased as a function of the attentional demands of the task, it was expected that the antisaccade cost would be greater for static targets than for dynamic targets. Second, we tested the proposal that offsets facilitate attentional disengagement. That is, antisaccade costs of onsets and offsets were compared in a variety of conditions.

\section{EXPERIMENT 1}

In Experiment 1, participants viewed displays consisting of two, three or four gray circles presented at six possible locations around a central fixation point. For one group of participants (the onset group) the search target was a new object that appeared at one of the unfilled locations. For another group of participants (the offset group) the search target was the offset of one of the circles, and for the final group of participants (the color singleton group) all but one of the objects turned red, leaving a gray color singleton target. Participants performed separate blocks of prosaccades and antisaccades in response to the search target. Note that the search target for the color singleton group was a static target (the only unchanged object), while the search target for the onset and offset groups was a dynamic target.

The goal of this experiment was to replicate the findings of Godijn and Kramer (2006) and Pratt and Trottier (2005) within a single experiment using a common method. Specifically, Godijn and Kramer showed that the antisaccade cost was greater for color singletons than for onsets, whereas Pratt and Trottier, using a different experimental set-up, showed that the antisaccade cost was greater for offsets than for onsets. The purpose of the display size manipulation was to prevent a confound of the number of objects in the fixation display or the target display between the onset, offset and color singleton groups.

\section{Method}

Participants. After giving their informed consent, twenty-four students from the University of Illinois with normal or corrected-tonormal vision served as paid volunteers.

Apparatus. A Pentium-based computer with a 21-in. color monitor controlled the timing of events and generated stimuli. Eye 


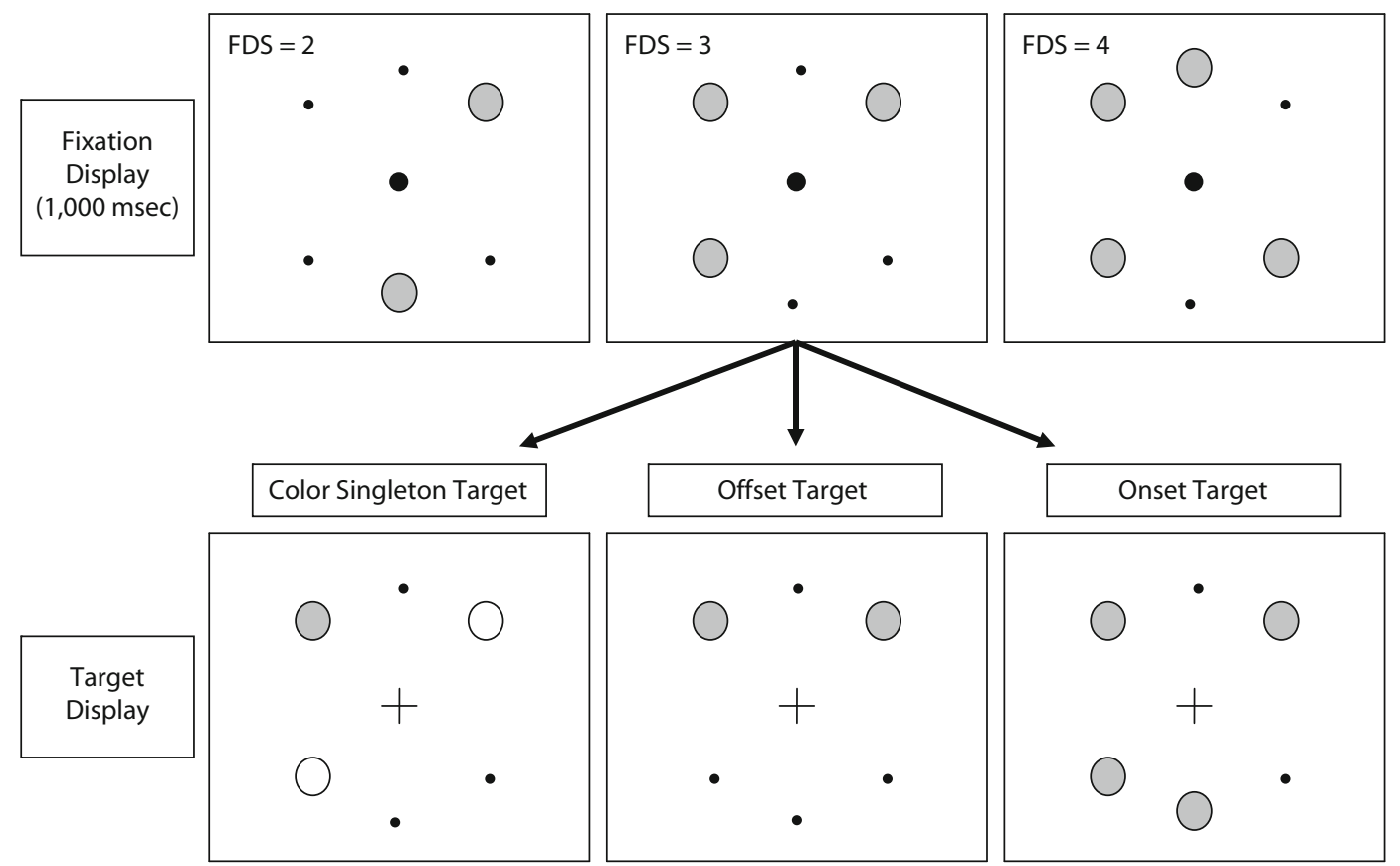

Figure 1. Examples of the displays in Experiment 1. Participants viewed fixation displays containing 2, 3 or 4 gray circles (FDS = fixation display size). Separate groups of participants searched for a color singleton target (bottom left panel), an offset target (bottom center panel) or an onset target (bottom right panel). Participants were required to execute a prosaccade or antisaccade in response to the target. See the text for further details.

movements were recorded by means of an Eyelink II tracker. An eye movement was considered a saccade when the velocity exceeded $35 \% \mathrm{sec}$ or the acceleration exceeded $9,500^{\circ} / \mathrm{sec}^{2}$. When participants were fixating the central fixation point at the start of each trial they pressed a key, which caused a recalibration of the participants' gaze point to the central fixation point. After this the trial started. Each participant was tested in a dimly lit room. They held their head on a chinrest, located $70 \mathrm{~cm}$ away from the monitor.

Stimuli, Procedure, and Design. At the start of each trial participants viewed displays (the fixation display) containing two, three or four gray circles $\left(1.2^{\circ}\right.$ in diameter), which were randomly presented at six possible object locations around a white central fixation point $\left(0.2^{\circ}\right)$ at an eccentricity of $7.6^{\circ}$ on a black background. Two of the six possible object locations were directly above and below the central fixation point and the four other possible object locations were the corner positions of an imaginary square around the fixation point. At the unfilled locations there were small white dots $\left(0.2^{\circ}\right)$. After $1,000 \mathrm{msec}$, the fixation dot changed into a fixation cross, signaling the onset of the search target display. For one group of participants the search target was a new gray circle which appeared at one of the unfilled locations (onset condition). The eight participants in this condition performed one block of onset-pro trials and one block of onset-anti trials. On onset-pro trials the task was to execute a saccade (a prosaccade) toward the onset location. On onset-anti trials the task was to execute a saccade (an antisaccade) to the location opposite the onset. For another group of participants the search target was the offset of one of the circles in the display (offset condition). The eight participants in this condition performed one block of offset-pro trials and one block of offset-anti trials. On offset-pro trials participants were required to execute a prosaccade toward the offset location. On offset-anti trials participants were required to execute an antisaccade away from the offset (to the location opposite the offset). For the remaining group of participants all but one of the circles turned red, leaving one gray color singleton target (the color singleton condition). The eight participants in this condition performed one block of color-pro trials and one block of color-anti trials. On color-pro tri- als the task was to execute a prosaccade toward the color singleton, while on color-anti trials the task was to execute an antisaccade away from the color singleton. See Figure 1 for examples of the stimulus display.

The search display was removed $500 \mathrm{msec}$ after the correct saccade. There were 360 trials in the prosaccade block and the antisaccade block and both blocks were preceded by 20 practice trials. The order of the blocks was randomized across participants.

\section{Results}

Discarded data. Trials on which the initial saccade latency was below $80 \mathrm{msec}(1.4 \%$ of trials) or above $1,000 \mathrm{msec}(0.1 \%$ of trials) were discarded from further analyses.

Initial saccade destination. The distance between the initial saccade endpoint and the six possible object locations was used to determine the initial saccade destination. The saccade was assigned to the position closest to the saccade endpoint. Table 1 shows the percentage of initial saccades that were directed to the prosaccade location, the antisaccade location and the other locations as a function of fixation display size and task for each group of participants. An ANOVA was conducted on the percentage of erroneous prosaccades in the antisaccade condition with fixation display size as within-subjects factor and search target (color singleton, onset, offset) as between-subjects factor. This revealed a main effect of search target $[F(2,21)=5.91, p<.01]$; participants searching for a color singleton executed more erroneous prosaccades than participants searching for an onset or offset and participants searching for an onset executed more erroneous prosaccades than those searching for an 
Table 1

Percentages of Initial Saccades to the Prosaccade Location, the Antisaccade Location, and Other Locations As a Function of Search Target, Fixation Display Size, and Task in Experiment 1

\begin{tabular}{|c|c|c|c|c|c|c|c|}
\hline \multirow{3}{*}{$\begin{array}{l}\text { Search } \\
\text { Target }\end{array}$} & \multirow{3}{*}{$\begin{array}{c}\text { Fixation } \\
\text { Display } \\
\text { Size }\end{array}$} & \multicolumn{6}{|c|}{ Location } \\
\hline & & \multicolumn{3}{|c|}{ Prosaccade Task } & \multicolumn{3}{|c|}{ Antisaccade Task } \\
\hline & & Prosaccade & Antisaccade & Other & Prosaccade & Antisaccade & Other \\
\hline \multirow[t]{3}{*}{ Color singleton } & 2 & 92.8 & 0.8 & 6.4 & 24.7 & 56.7 & 18.6 \\
\hline & 3 & 90.6 & 1.6 & 7.8 & 30.8 & 53.9 & 15.4 \\
\hline & 4 & 91.0 & 1.6 & 7.4 & 36.0 & 53.9 & 10.1 \\
\hline \multirow[t]{3}{*}{ Onset } & 2 & 98.3 & 0.8 & 0.9 & 21.4 & 69.4 & 9.2 \\
\hline & 3 & 97.4 & 1.3 & 1.4 & 19.8 & 73.0 & 7.2 \\
\hline & 4 & 98.5 & 0.4 & 1.1 & 17.7 & 75.5 & 6.9 \\
\hline \multirow[t]{3}{*}{ Offset } & 2 & 96.5 & 0.6 & 2.9 & 5.7 & 81.4 & 13.0 \\
\hline & 3 & 95.8 & 1.3 & 2.9 & 7.1 & 76.3 & 16.6 \\
\hline & 4 & 95.4 & 1.6 & 3.1 & 6.3 & 75.8 & 17.9 \\
\hline
\end{tabular}

offset. There was a main effect of fixation display size $[F(2,42)=3.73, p<.04]$, reflecting an increase in percentage of erroneous prosaccades as a function of fixation display size. There was also an interaction between search target and display size $[F(4,42)=9.10, p<$ $.001]$. Whereas the percentage of erroneous prosaccades increased as a function of fixation display size for participants searching for a color singleton target $[F(2,14)=$ $13.22, p<.001]$, there was no significant effect of display size on the percentage of erroneous prosaccades for participants searching for an onset $[F(2,14)=1.81, p>$ $.05]$, or an offset $[F(2,14)<1]$.

Saccade latency. The mean latencies of correct prosaccades, antisaccades as well as the antisaccade cost (difference in correct saccade latencies between the prosaccade and antisaccade conditions) are shown for each condition in Table 2. An ANOVA with task (prosaccade or antisaccade) and fixation display size (2, 3 or 4$)$ as within-subjects factors and search target as betweensubjects factor was conducted on the mean latencies of the correct saccades. A main effect of search target was found $[F(2,21)=7.55, p<.01]$. Saccade latencies were longer for participants searching for a color singleton than for those searching for an offset and they were longer for participants searching for an offset than for those searching for an onset. There was also a main effect of task $[F(1,21)=362.17, p<.001]$, indicating that correct prosaccade latencies were shorter than correct antisaccade latencies. There was no significant main effect of fixation display size $[F(2,42)<1]$. An interaction was found between search target and task $[F(2,21)=40.77, p<.001]$, revealing that there was a difference in antisaccade cost between the groups. Separate ANOVAs confirmed that the antisaccade cost was greater for participants searching for a color singleton (antisaccade cost: $140 \mathrm{msec}$ ) than for those searching for an onset (antisaccade cost: $99 \mathrm{msec}$ ) $[F(1,14)=10.19, p<.01]$. Furthermore, the antisaccade cost was greater for participants searching for an onset than for those searching for an offset (antisaccade cost: $35 \mathrm{msec})[F(1,14)=28.11, p<.001]$. There was also an interaction between fixation display size and search target $[F(4,42)=3.57, p<.03]$. A significant reduction in saccade latency as a function of display size was found for an onset target $[F(2,14)=19.04, p<.001]$, but no significant effect of display size on saccade latencies was found for a color singleton target $[F(2,14)<1]$, or an offset target $[F(2,14)=2.85, p>.05]$.

\section{Discussion}

The results of Experiment 1 replicated the main results of Godijn and Kramer (2006) and Pratt and Trottier (2005). Specifically, the antisaccade cost was greater for color singletons than for onsets and it was greater for onsets than for offsets. Furthermore, there were more er-

Table 2

Latency of Correct Saccades (in Milliseconds), and Standard Errors, in the Prosaccade and Antisaccade Tasks, and Antisaccade Cost, As a Function of Search Target and Fixation Display Size in Experiment 1

\begin{tabular}{|c|c|c|c|c|c|c|}
\hline \multirow{3}{*}{$\begin{array}{l}\text { Search } \\
\text { Target }\end{array}$} & \multirow{3}{*}{$\begin{array}{c}\text { Fixation } \\
\text { Display } \\
\text { Size }\end{array}$} & \multicolumn{4}{|c|}{ Task } & \multirow{3}{*}{$\begin{array}{c}\text { Antisaccade } \\
\text { Cost }\end{array}$} \\
\hline & & \multicolumn{2}{|c|}{ Prosaccade } & \multicolumn{2}{|c|}{ Antisaccade } & \\
\hline & & RT & $S E$ & RT & $S E$ & \\
\hline \multirow[t]{3}{*}{ Color singleton } & 2 & 253 & 7.4 & 385 & 12.8 & 132 \\
\hline & 3 & 253 & 7.1 & 397 & 11.2 & 144 \\
\hline & 4 & 255 & 6.7 & 400 & 15.3 & 145 \\
\hline \multirow[t]{3}{*}{ Onset } & 2 & 214 & 10.2 & 320 & 17.4 & 106 \\
\hline & 3 & 213 & 11.3 & 310 & 18.8 & 97 \\
\hline & 4 & 207 & 10.2 & 302 & 19.8 & 95 \\
\hline \multirow[t]{3}{*}{ Offset } & 2 & 250 & 14.0 & 291 & 10.0 & 41 \\
\hline & 3 & 259 & 15.8 & 288 & 8.9 & 29 \\
\hline & 4 & 260 & 18.0 & 294 & 11.9 & 34 \\
\hline
\end{tabular}

Note-Antisaccade cost is the difference in latency between correct antisaccades and correct prosaccades. 
Table 3

Percentages of Initial Saccades to the Prosaccade Location, the Antisaccade Location, and Other Locations As a Function of Onset Presentation Duration (in Milliseconds) and Task in Experiment 2

\begin{tabular}{|c|c|c|c|c|c|c|}
\hline \multirow{3}{*}{$\begin{array}{c} \\
\text { Onset } \\
\text { Presentation } \\
\text { Duration }\end{array}$} & \multicolumn{6}{|c|}{ Location } \\
\hline & \multicolumn{3}{|c|}{ Prosaccade Task } & \multicolumn{3}{|c|}{ Antisaccade Task } \\
\hline & Prosaccade & Antisaccade & Other & Prosaccade & Antisaccade & Other \\
\hline 50 & 90.2 & 3.6 & 6.2 & 8.6 & 74.2 & 17.2 \\
\hline 100 & 91.1 & 3.2 & 5.7 & 11.1 & 72.8 & 16.1 \\
\hline 150 & 91.5 & 2.7 & 5.9 & 15.0 & 68.9 & 16.2 \\
\hline 200 & 93.4 & 1.7 & 5.0 & 16.9 & 69.0 & 14.1 \\
\hline 250 & 93.6 & 1.9 & 4.4 & 18.9 & 66.4 & 14.7 \\
\hline
\end{tabular}

roneous prosaccades toward color singletons than toward onsets and there were more erroneous prosaccades toward onsets than toward offsets. These results suggest a relationship between erroneous prosaccades and antisaccade cost; the greater the antisaccade cost the larger the proportion of erroneous prosaccades, at least with the search targets used in the present experiment (onsets, offsets and color singletons). This pattern of results is consistent with Godijn and Kramer (2006) and Pratt and Trottier (2005).

The finding of a greater antisaccade cost with onsets than with offsets seems contrary to our proposal that an increase in attentional demands increases antisaccade cost. However, both onset and offset targets are dynamic and the attentional demands are expected to be limited. We suggest that the difference in antisaccade cost between onsets and offsets is the result of a faster disengagement of attention from the prosaccade location with offsets than with onsets.

\section{EXPERIMENT 2}

The goal of Experiment 2 was to examine whether the offset of the search target reduces the antisaccade cost by facilitating the disengagement of attention at the location of the search target. Participants searched for an onset target, which was removed after a variable presentation duration. In separate blocks participants were required to execute prosaccades or antisaccades in response to the onset target. It was expected that the antisaccade cost would increase as a function of presentation duration.

\section{Method}

Participants. After giving their informed consent, 16 students of the University of Illinois with normal or corrected-to-normal vision served as paid volunteers.

Stimuli, Procedure, and Design. The stimuli were the same as in the onset condition of Experiment 1 with two exceptions: First, there were always three gray circles randomly presented at three of the six possible locations. Second, after the presentation of the onset at one of the unfilled locations, the onset circle was removed and replaced by a small white dot after a variable delay. The onset was removed 50,100,150,200, or $250 \mathrm{msec}$ after its presentation. Participants performed two types of trials: On onset-pro trials they were required to execute a prosaccade toward the onset, while on onset-anti trials they were required to execute an antisaccade away from the onset. Half of the participants started with the prosaccade block, the other half started with the antisaccade block. The presentation duration of the onset was randomized within blocks. There were 300 trials per block and each block was preceded by a practice block of 20 trials.

\section{Results}

Discarded data. Trials on which the initial saccade latency was below $80 \mathrm{msec}$ (3.7\% of trials) or above $1,000 \mathrm{msec}(0.2 \%$ of trials $)$ were discarded from further analyses.

Initial saccade destination. Table 3 shows the percentage of initial saccades that were directed to the prosaccade location, the antisaccade location and the other locations as a function of onset presentation duration and task. An ANOVA on the percentage of erroneous prosaccades with onset presentation duration $(50,100,150,200$, or $250 \mathrm{msec})$ as factor revealed a significant effect of onset presentation duration $[F(4,60)=12.62, p<.001]$. As can be seen in Table 3 the percentage of erroneous prosaccades increased as a function of onset presentation duration.

Saccade latency. Table 4 shows the mean latencies of correct prosaccades, antisaccades and the antisaccade cost as a function of onset presentation duration. An ANOVA with task (prosaccade or antisaccade) and onset presentation duration as within-subjects factors was conducted on the mean latencies of the correct saccades. There was a main effect of task $[F(1,15)=69.07, p<.001]$, indicating that correct saccade latencies were longer in the antisaccade task than in the prosaccade task. The main effect of onset presentation duration failed to reach significance $[F(4,60)=2.46, p>.05]$, but there was a significant interaction between onset presentation duration and task $[F(4,60)=13.75, p<.001]$. Specifically, the antisaccade cost increased as a function of onset presenta-

Table 4

Latency of Correct Saccades (in Milliseconds), and Standard Errors, in the Prosaccade and Antisaccade Tasks, and Antisaccade Cost, As a Function of Onset Presentation Duration (in Milliseconds) in Experiment 2

\begin{tabular}{|c|c|c|c|c|c|}
\hline \multirow{3}{*}{$\begin{array}{l}\text { Onset } \\
\text { Presentation } \\
\text { Duration }\end{array}$} & \multicolumn{4}{|c|}{ Task } & \multirow{3}{*}{$\begin{array}{c}\text { Antisaccade } \\
\text { Cost }\end{array}$} \\
\hline & \multicolumn{2}{|c|}{ Prosaccade } & \multicolumn{2}{|c|}{ Antisaccade } & \\
\hline & RT & $\overline{S E}$ & $\overline{\mathrm{RT}}$ & $\overline{S E}$ & \\
\hline 50 & 241 & 9.6 & 303 & 11.0 & 62 \\
\hline 100 & 234 & 10.2 & 305 & 10.9 & 71 \\
\hline 150 & 217 & 6.7 & 313 & 14.4 & 96 \\
\hline 200 & 215 & 6.7 & 311 & 12.0 & 96 \\
\hline 250 & 218 & 6.0 & 320 & 14.1 & 102 \\
\hline
\end{tabular}




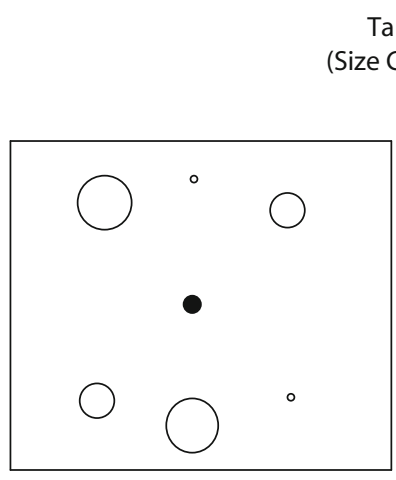

Fixation Display
Target

Size Change)

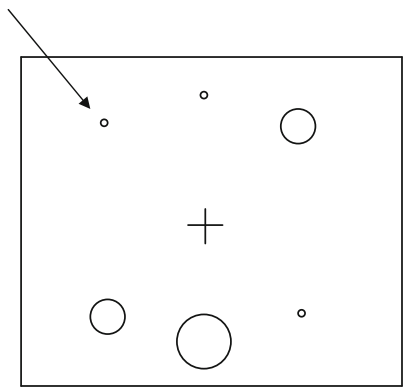

Target Display
Figure 2. Example of the display sequence in Experiment 3. Shown is a 'large-small' trial, in which the size of the target circle changes from large to small.

tion duration. Further ANOVAs revealed that prosaccade latencies decreased as a function of presentation duration $[F(4,60)=15.10, p<.001]$, while antisaccade latencies increased as a function of presentation duration $[F(4,60)=3.62, p<.01]$.

\section{Discussion}

The results of Experiment 2 were consistent with the hypothesis that the offset of the search target facilitates antisaccade performance; the antisaccade cost increased as a function of presentation duration of the onset target. Presentation duration affected both prosaccade and antisaccade latencies; prosaccade latencies decreased as a function of presentation duration while antisaccade latencies increased as a function of presentation duration. It is possible that the effect on prosaccade latencies was due to the disruption of prosaccade programming when the offset of the target occurred early in time. Alternatively, the prosaccade effect could be explained as a result of interference of the localization of the target. That is, the interference could be related to target localization as opposed to prosaccade programming. A final possibility is that the offset of the target facilitates disengagement of attention from the prosaccade location. It is plausible that attentional disengagement interferes with the generation of prosaccades. These three possibilities do not necessarily exclude one another given the likelihood that these processes are related. In any case, the reduction in antisaccade latency as a function of presentation duration still needs to be explained. Furthermore, the reduction in antisaccade latency goes together with an increase in erroneous prosaccades as a function of presentation duration in the antisaccade task. These two effects are best explained by the idea that the offset of the target facilitates disengagement of attention from the prosaccade location. This allows a fast shift of attention to the antisaccade location and reduces antisaccade latency. Also note that the antisaccade costs in Experiment 2 are between the offset and onset antisaccade costs of Experiment 1. The antisaccade costs in Experiment 2 increase as a function of presentation duration from about $60 \mathrm{msec}$ to about
$100 \mathrm{msec}$, while the onset antisaccade cost of Experiment 1 was around $100 \mathrm{msec}$. Thus, the later the offset of the target occurs the greater the probability that attention has already disengaged from the prosaccade location, rendering the offset ineffective.

Although the results of Experiment 1 and 2 suggest that disengagement of attention is faster with offsets than with onsets, it is yet unclear whether it is the change in the object that is the critical factor or the presence of a large object at the target location. That is, the dynamic nature of the target (onset vs. offset) is confounded with the size of the object at the prosaccade location.

\section{EXPERIMENT 3}

The purpose of Experiment 3 was to examine whether the antisaccade cost is affected by the dynamic nature (onset vs. offset) or the size of the target at the prosaccade location. Fixation displays consisted of two large-sized circles, two medium-sized circles and two small-sized circles. The search target was a change (onset or offset) in the size of one of the objects. This resulted in three types of onsets (smallmedium, small-large and medium-large) and three types of offsets (medium-small, large-small and large-medium). Note that in the small-medium and the large-medium conditions the target size is identical, but there was a difference in the dynamic nature of the target (onset vs. offset).

\section{Method}

Participants. After giving their informed consent twelve students of the University of Illinois with normal or corrected-to-normal vision served as paid volunteers.

Stimuli, Procedure, and Design. At the start of each trial participants viewed displays (the fixation display) containing white circles, positioned around a central fixation point. The position of the circles was identical to the previous experiments. Two of the circles (the large-sized circles) had a diameter of $1.29^{\circ}$, two (the medium-sized circles) had a diameter of $0.92^{\circ}$, and two (the small-sized circles) had a diameter of $0.18^{\circ}$. The size difference between the large-sized circle and the medium-sized circle was approximately the same as the size difference between the medium-sized circle and the smallsized circle. The position of these circles was randomized within blocks. After 1,000 msec, the search target was defined as a change in the size of one of the circles. That is, the target was the circle that changed into one of the two other sizes. Thus, there were three types of onset targets: small-medium (in which a small-sized circle changed into a medium-sized circle), small-large, medium-large, and three types of offset targets: medium-small, large-medium, and large-small. See Figure 2 for an example of the display sequence. These dynamic (onset and offset) target types were randomized within blocks. Participants performed two blocks of 288 trials. In one block participants were instructed to execute a prosaccade toward the change, in the other block they were instructed to execute an antisaccade away from the change. The order of the blocks was randomized across participants.

\section{Results}

Discarded data. Trials on which the initial saccade latency was below $80 \mathrm{msec}(3.7 \%$ of trials) or above $1,000 \mathrm{msec}(0.2 \%$ of trials $)$ were discarded from further analyses.

Initial saccade destination. Table 5 shows the percentage of initial saccades that were directed to the 
Table 5

Percentages of Initial Saccades to the Prosaccade Location, the Antisaccade Location, and Other Locations As a Function of Dynamic Target Type and Task in Experiment 3

\begin{tabular}{|c|c|c|c|c|c|c|c|}
\hline \multirow{2}{*}{$\begin{array}{c}\text { Dynamic Target } \\
\text { Type }\end{array}$} & \multirow{2}{*}{$\begin{array}{l}\text { Onset/ } \\
\text { Offset }\end{array}$} & \multicolumn{3}{|c|}{ Prosaccade Task } & \multicolumn{3}{|c|}{ Antisaccade Task } \\
\hline & & Prosaccade & Antisaccade & Other & Prosaccade & Antisaccade & Other \\
\hline Small-medium & onset & 98.0 & 1.0 & 1.0 & 15.4 & 77.9 & 6.8 \\
\hline Small-large & onset & 99.2 & 0.3 & 0.5 & 16.2 & 77.4 & 6.5 \\
\hline Medium-large & onset & 98.8 & 0.8 & 0.3 & 13.4 & 79.9 & 6.6 \\
\hline Medium-small & offset & 97.3 & 1.7 & 1.0 & 7.1 & 83.3 & 9.6 \\
\hline Large-small & offset & 97.6 & 1.2 & 1.2 & 4.9 & 85.8 & 9.4 \\
\hline Large-medium & offset & 98.5 & 1.0 & 0.5 & 8.2 & 80.8 & 11.0 \\
\hline
\end{tabular}

prosaccade location, the antisaccade location and the other locations as a function of dynamic target type and task. An ANOVA on the percentage of erroneous prosaccades revealed a significant effect of dynamic target type $[F(5,55)=5.88, p<.001]$. A planned comparison showed a significant difference in percentage of prosaccade errors between onset and offset targets (averaged across target types) $[t(11)=3.12, p<.01]$. However, the difference in percentage of prosaccade errors between the small-medium and large-medium target types (the only comparison of an onset with an offset, in which the target size is constant) failed to reach significance $[t(11)=1.79, p>.10]$.

Saccade latency. Table 6 shows the mean latencies of correct prosaccades, antisaccades and the antisaccade cost as a function of dynamic target type. An ANOVA with task (prosaccade or antisaccade) and dynamic target type as within-subjects factors was conducted on the mean latencies of the correct saccades. There was a main effect of task $[F(1,11)=30.33, p<.001]$, a main effect of dynamic target type $[F(5,55)=27.15, p<.001]$, and an interaction between task and dynamic target type $[F(5,55)=11.62$, $p<.001]$, revealing a difference in antisaccade cost between dynamic target types. To examine whether there was a significant difference between onset and offset target types an additional ANOVA was conducted in which mean saccade latencies were averaged across target types for both onset and offset targets. A main effect of target type $[F(1,11)=60.42, p<.001]$, indicated that saccade latencies were longer with offset targets than onset targets. Furthermore, an interaction between target type and task $[F(1,11)=40.82, p<.001]$, revealed that the antisaccade cost was greater with offset targets than with onset targets. Finally, to examine whether the antisaccade cost differed between onset and offset targets when the target size was identical, we conducted a separate ANOVA with only the small-medium and large-medium target types. A significant interaction was found between target type and task $[F(1,11)=23.57, p<.001]$, indicating a difference in antisaccade cost between onsets and offsets even when the size of the target is identical.

\section{Discussion}

The results of Experiment 3 demonstrated a greater antisaccade cost with onset targets than with offset targets, irrespective of the size of the object at the prosaccade location. That is, the dynamic nature of the target was the critical factor in determining the antisaccade cost, while the initial and altered sizes of the target did not have much effect. Furthermore, the proportion of erroneous prosaccades was greater with onset targets than with offset targets.

Taken together the findings of the first three experiments provide evidence for the idea that offsets facilitate disengagement of attention, thereby reducing the antisaccade cost. The antisaccade costs of these dynamic targets ranged from around $35 \mathrm{msec}$ (offsets in Experiment 1) to around $100 \mathrm{msec}$ (onsets in Experiment 1 and Experiment 2). In contrast, when a static target was employed in Experiment 1, through a condition in which the target was defined as the only circle that did not change color, an antisaccade cost of around $140 \mathrm{msec}$ was found. This suggests that the antisaccade cost may be greater with static targets than with dynamic target. However, the static or dynamic nature of the target is not the only difference between the color singleton target of Experiment 1 and the onset and offset targets of the first three experiments. Most notably, the color singleton target was not just the only unchanged object, it was also a uniquely colored object in the target display.

\section{EXPERIMENT 4}

The goal of Experiment 4 was to examine the antisaccade cost with dynamic and static targets. Specifically, the display sequences were designed such that a given target display could arise through the change in the target object, or the nontarget objects. That is, for each target/nontarget arrangement there was a condition with a static target (but dynamic nontargets) and a condition with a dynamic target (but static nontargets).

Table 6

Latency of Correct Saccades (in Milliseconds), and Standard Errors, in the Prosaccade and Antisaccade Tasks, and Antisaccade Cost, As a Function of Dynamic Target Type in Experiment 3

\begin{tabular}{|c|c|c|c|c|c|c|}
\hline \multirow{3}{*}{$\begin{array}{c}\text { Dynamic Target } \\
\text { Type }\end{array}$} & \multirow{3}{*}{$\begin{array}{l}\text { Onset/ } \\
\text { Offset }\end{array}$} & \multicolumn{4}{|c|}{ Task } & \multirow{3}{*}{$\begin{array}{c}\text { Antisaccade } \\
\text { Cost }\end{array}$} \\
\hline & & \multicolumn{2}{|c|}{ Prosaccade } & \multicolumn{2}{|c|}{ Antisaccade } & \\
\hline & & RT & $S E$ & $\mathrm{RT}$ & $S E$ & \\
\hline Small-medium & onset & 217 & 5.0 & 288 & 13.6 & 71 \\
\hline Small-large & onset & 212 & 3.6 & 289 & 13.9 & 77 \\
\hline Medium-large & onset & 221 & 5.9 & 298 & 14.4 & 77 \\
\hline Medium-small & offset & 256 & 8.6 & 306 & 15.3 & 50 \\
\hline Large-small & offset & 246 & 8.7 & 298 & 13.3 & 52 \\
\hline Large--medium & offset & 242 & 4.8 & 296 & 15.4 & 54 \\
\hline
\end{tabular}




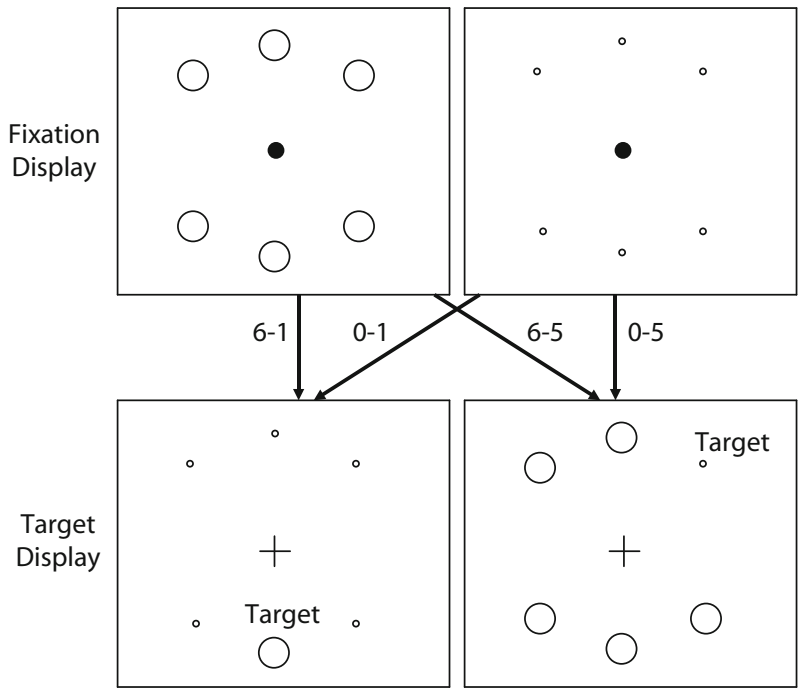

Figure 3. Examples of the display sequence in Experiment 4. The fixation display consists of six circles or six dots and the target display consists of five circles and a single dot or five dots and a single circle. The labels of each display sequence refer to the change in the number of circles.

\section{Method}

Participants. After giving their informed consent eight students of the University of Illinois with normal or corrected-to-normal vision served as paid volunteers.

Stimuli, Procedure, and Design. At the start of each trial participants viewed one of two possible fixation displays. On half of the trials there were six white dots $\left(0.2^{\circ}\right.$ in diameter) positioned around the central fixation point. On the other half of the trials there were six circles $\left(1.2^{\circ}\right.$ in diameter) positioned around the central fixation point. The six positions were identical to the previous experiments. After 1,000 msec, the target display was presented. On half the trials the target display consisted of five circles and one dot, on the other half of the trials it consisted of five dots and one circle. Thus, there were four types of trials, which are labeled according to the change in the number of circles between the fixation display and the target display: On "six-one" trials, five of the six circles were replaced by dots; on "six-five" trials, one of the six circles was replaced by a dot; on "zero-one" trials, one of the dots was replaced by a circle; and on "zero-five" trials, five of the six dots were replaced by circles. See Figure 3 for examples of the display sequence. Participants performed four blocks of 144 trials. In two blocks participants were instructed to execute a prosaccade toward the unique peripheral element (the only circle or the only dot). In the two other blocks they were instructed to execute an antisaccade away from the unique peripheral element. Note that in two conditions, the "zero-five" and the "six-one" conditions, the search target was static (no change at the location of the search target), while in the two other conditions, the "zero-one" and the "six-five" conditions, the search target was dynamic (an onset or offset at the search target location). These conditions and the location of the target were randomized within blocks. The order of the blocks was counterbalanced across participants.

\section{Results}

Discarded data. Trials on which the initial saccade latency was below $80 \mathrm{msec}(1.6 \%$ of trials) or above $1,000 \mathrm{msec}(0.1 \%$ of trials $)$ were discarded from further analyses.

Initial saccade destination. Table 7 shows the percentage of initial saccades that were directed to the prosaccade location, the antisaccade location and the other locations as a function of condition and task. An ANOVA was conducted on the percentage of erroneous prosaccades with target object (dot vs. circle) and target type (dynamic vs. static) as factors. The main effects of target object and target type failed to reach significance $[F(1,7)=3.77$, $p>.05$, and $F(1,7)=2.96, p>.10$, respectively]. However, we also conducted an ANOVA on the percentage of saccades to 'other' locations (not the prosaccade or antisaccade location) and found a main effect of target type $[F(1,7)=30.71, p<.001]$. There were significantly more saccade errors to 'other' locations with static targets than with dynamic targets.

Saccade latency. Table 8 shows the mean latencies of correct prosaccades, antisaccades and the antisaccade cost as a function of condition. An ANOVA with task (prosaccade or antisaccade), target object (dot or circle) and target type (dynamic or static) as within-subjects factors was conducted on the mean latencies of the correct saccades. There was a main effect of task $[F(1,7)=79.87$, $p<.001]$, a main effect of target object $[F(1,7)=13.40$, $p<.01]$, and a main effect of target type $[F(1,7)=$ $106.22, p<.001]$. There was also a significant interaction between task and target type $[F(1,7)=8.55, p<.025]$, revealing a greater antisaccade cost for static targets than for dynamic targets. Finally, a significant interaction was found between target type and target object $[F(1,7)=$ $16.12, p<.01]$.

\section{Discussion}

The main finding of Experiment 4 is that the antisaccade cost was greater with static targets than with dynamic targets. For both of the possible target/nontarget arrangements, the antisaccade cost was greater when the nontargets changed than when the target changed. Although there was no significant effect on erroneous prosaccades, there

Table 7

Percentages of Initial Saccades to the Prosaccade Location, the Antisaccade Location, and Other Locations As a Function of Condition and Task in Experiment 4

\begin{tabular}{|c|c|c|c|c|c|c|c|c|}
\hline \multirow[b]{3}{*}{ Condition } & \multirow{3}{*}{$\begin{array}{l}\text { Target } \\
\text { Object }\end{array}$} & \multirow[b]{3}{*}{ Target Type } & \multicolumn{6}{|c|}{ Location } \\
\hline & & & \multicolumn{3}{|c|}{ Prosaccade Task } & \multicolumn{3}{|c|}{ Antisaccade Task } \\
\hline & & & Prosaccade & Antisaccade & Other & Prosaccade & Antisaccade & Other \\
\hline Zero-one & circle & dynamic (onset) & 97.7 & 0.4 & 1.9 & 19.0 & 74.9 & 6.2 \\
\hline Zero-five & dot & static & 78.7 & 2.5 & 18.8 & 18.4 & 50.8 & 30.8 \\
\hline Six-one & circle & static & 83.7 & 2.7 & 13.7 & 25.5 & 45.3 & 29.3 \\
\hline Six-five & dot & dynamic (offset) & 97.3 & 0.5 & 2.2 & 11.2 & 79.0 & 9.8 \\
\hline
\end{tabular}


Table 8

Latency of Correct Saccades (in Milliseconds), and Standard Errors, in the Prosaccade and Antisaccade Tasks, and Antisaccade Cost, As a Function of Condition in Experiment 4

\begin{tabular}{lllllllr}
\hline & & & \multicolumn{4}{c}{ Task } \\
\cline { 3 - 6 } & Target & & \multicolumn{2}{c}{ Prosaccade } & \multicolumn{2}{c}{ Antisaccade } & Antisaccade \\
\cline { 3 - 6 } Condition & Object & Target Type & RT & $S E$ & RT & $S E$ & Cost \\
\hline Zero-one & circle & dynamic (onset) & 197 & 6.3 & 271 & 14.0 & 74 \\
Zero-five & dot & static & 269 & 9.9 & 378 & 17.3 & 109 \\
Six-one & circle & static & 269 & 11.9 & 382 & 24.9 & 113 \\
Six-five & dot & dynamic (offset) & 242 & 12.0 & 324 & 21.0 & 82 \\
\hline
\end{tabular}

were significantly more erroneous saccades to 'other' (non prosaccade) locations in the static target conditions than in the dynamic target conditions. However, the approximately two-to-three ratio of prosaccade errors to 'other' errors in the static target conditions (which at chance level should be a ratio of one-to-four), suggests that the task-relevance of these static targets elicited erroneous prosaccades even though attention was likely to be captured by the dynamic nontargets. Similar to Experiment 1, in which the color singleton elicited frequent erroneous prosaccades, it is clear that these prosaccades are not reflexive or stimulus-driven.

\section{GENERAL DISCUSSION}

The present study examined the antisaccade costs (difference in latency between prosaccades and antisaccades) with dynamic (onsets and offsets) and static targets. The first main finding was that the antisaccade cost was greater for static targets than for dynamic targets. In Experiment 1 the antisaccade cost was greater for static color singletons than for onsets and offsets. Furthermore, in Experiment 4 the antisaccade cost was greater when the target location was the only location without an onset or offset than when it was the only location with an onset or offset. The second main finding was that, irrespective of the size of the target object, antisaccade costs were greater with offsets than with onsets. Moreover, the antisaccade cost was reduced when there was an offset of the onset target shortly after its appearance. Throughout the series of experiments the proportion of erroneous prosaccades typically followed the antisaccade cost. That is, there were more erroneous prosaccades to static targets than dynamic targets (except in Experiment 4, although there were more saccade errors to 'other' locations with static targets than with dynamic targets), there were more erroneous prosaccades with onset targets than with offset targets, and an early target offset reduced the proportion of erroneous prosaccades.

\section{Attentional Demands and Working Memory}

We propose that the greater antisaccade cost with static targets is the result of interference of working memory processes. As discussed in the Introduction several lines of research have illuminated the role of working memory in the antisaccade task (e.g., Kane et al., 2001; Kramer et al., 2005; Roberts et al., 1994; Unsworth et al., 2004; Walker, Husain, Hodgson, Harrison, \& Kennard, 1998). That is, in order to generate an antisaccade observers must maintain in their working memory the characteristics of the target, and especially the instruction to execute a saccade away from the target as opposed to the natural response of a saccade toward the target. When there is interference of working memory processes performance may be disrupted by what Duncan (1995) termed "goal neglect." That is, the goal of executing a saccade away from the target may not be maintained or it may not be applied to a specific situation. Alternatively, instead of a complete neglect of the goal there might be a delay in its application. Thus, when there is interference of working memory processes a greater proportion of erroneous prosaccades are expected as well as an increase in the antisaccade cost. The results of the present study suggest that the attentional demands of the search task interfere with working memory processes and increase the antisaccade cost as well as the proportion of erroneous prosaccades. Specifically, it was found that the antisaccade cost was greater with static targets than with dynamic targets. Furthermore, in Experiment 1 there were more erroneous prosaccades to the static color singleton than toward the dynamic targets. Although the difference in proportion of erroneous prosaccades between the static and dynamic targets in Experiment 4 did not reach significance, it is clear that the proportion of erroneous prosaccades with static targets surpassed what would have been expected by chance alone. Furthermore, this nonsignificant result is hard to interpret given the difference in proportion of erroneous saccades to "other" locations between static and dynamic targets.

The question remains why attentional demands of the target search should affect working memory processes. Engle and colleagues (e.g., Engle, 2000; Engle et al., 1999; Kane et al., 2001; Kane \& Engle, 2003; Kane et al., 2006; Unsworth et al., 2004) have proposed a controlledattention view of working memory capacity, according to which working memory capacity refers to the ability to effectively maintain stimulus representations, action plans, and goals in the face of interference. Furthermore, a strong interdependence between maintaining task goals in working memory and the control of attention is assumed. Similarly, we propose that the attentional control of the search task is guided by working memory processes (i.e., the identity of the target and other characteristics of the target display) and that if these attentional demands are great they interfere with the maintenance or application of other working memory processes. In the antisaccade task the attentional search for the target may interfere with the maintenance of the goal to saccade away from 
the target. It is likely that the maintenance and application of the antisaccade goal in working memory is particularly sensitive to interference given its unnatural nature and the fact that a more natural 'default' response (the prosaccade) is readily available. Indeed, a recent study by Kane et al. (2006) revealed that participants with a low working memory capacity were not impaired in a number of visual search tasks with varying attentional demands. Although further research is required to determine the conditions under which attentional demands interfere with maintenance of task goals in working memory, it is possible that the nature of the stimulus-response coupling is an important factor. That is, task goals related to unnatural responses that go against prepotent, or natural responses, are likely to be most sensitive to interference.

\section{Offsets and Attentional Disengagement}

It may be assumed that the attentional demands of searching for dynamic targets, such as onsets and offsets, is quite limited (at least compared to static targets). Nevertheless, we did find a significant difference in performance between onset and offset targets. The antisaccade cost is greater for onset targets than offset targets and the former also elicit more erroneous prosaccades (but still less than static targets). The results of the present study indicate that offsets facilitate disengagement of attention from the prosaccade location. Specifically, Experiment 2 revealed that the antisaccade cost was reduced and the proportion of erroneous prosaccades was increased as a function of presentation duration of the target. Prosaccade latencies were reduced as a function of presentation duration, while antisaccade latencies were increased. This is consistent with the idea that offsets facilitate disengagement of attention. Given the evidence for a strong relationship between attention and saccades (e.g., Deubel \& Schneider, 1996; Godijn \& Pratt, 2002; Hoffman \& Subramaniam, 1995; Kowler, Anderson, Dosher, \& Blaser, 1995) it is plausible that a facilitated disengagement of attention interferes with the programming of a prosaccade (thereby reducing the proportion of erroneous prosaccades and increasing correct prosaccade latencies) and allows a fast shift of attention to the antisaccade location, reducing the antisaccade latency.

\section{Working Memory and Attentional Control}

We propose a model of antisaccade production, in which attentional control and working memory play an important role. Specifically, attention shifts serially from the prosaccade to the antisaccade location. That is, attention is allocated to the target in order to localize it, after which attention is disengaged from the target and shifted to the antisaccade location prior to the execution of the antisaccade. This attentional control is guided by working memory processes related to the goals that are relevant for the task (i.e., the identity of the target, other relevant characteristics of the search task, and the goal of executing a saccade away from the target).

The idea that attention shifts from the prosaccade location to the antisaccade location is consistent with evidence that attention is required in order to program voluntary saccades (see, e.g., Deubel \& Schneider, 1996; Godijn \& Pratt, 2002; Hoffman \& Subramaniam, 1995; Kowler et al., 1995). Electrophysiological evidence that attention is shifted from the target location to the antisaccade location was provided by Everling, Spantekow, Krappmann and Flohr (1998). Their results revealed a negative eventrelated potential after presentation of the target stimulus, which was maximal over the contralateral parietal cortex. During programming of the antisaccade this negative potential was reduced over the contralateral parietal hemisphere and increased over the parietal hemisphere ipsilateral to the stimulus. This finding provides evidence that attention shifts from the target location to the antisaccade location, since there is abundant evidence that the parietal cortex is involved in spatial shifts of attention (e.g., Goldberg, Bisley, Powell, Gottlieb, \& Kusonoki, 2002; Lynch, Mountcastle, Talbot, \& Yin, 1977; Yantis et al., 2002).

Within the framework of our proposed model the present findings demonstrate two ways in which the shift of attention from the prosaccade location to the antisaccade location can be affected. One of these is endogenous, the other exogenous. The endogenous factor is the degree to which working memory processes related to the goal of executing a saccade away from the target can be maintained and applied on a given trial. Specifically, the attentional demands of the search task can interfere with these working memory processes. The exogenous factor is the offset at the target location, which facilitates disengagement of attention from the prosaccade location. The following sections address how this model compares with previous views of antisaccade generation, in which reflexive prosaccade programming and inhibition play an important role.

\section{Reflexive Prosaccade Programming}

Traditional accounts of antisaccade generation have assumed that in the antisaccade task a reflexive prosaccade is automatically programmed in response to the onset of the target (e.g., Guitton et al., 1985; Hallett, 1978; Hallett \& Adams, 1980). According to this view an antisaccade can only be executed if the reflexive prosaccade is first inhibited (or canceled). Thus, erroneous prosaccades are executed if the reflexive saccade program is not inhibited before it runs to completion. For example, when Guitton et al. discovered that patients with frontal lesions executed a higher proportion of erroneous prosaccades (also see Pierrot-Deseilligny et al., 2002; Pierrot-Deseilligny et al., 1991; Milea et al., 2003) they interpreted this as evidence that in frontal patients the cancellation signal is delayed. However, since frontal areas are also involved in working memory the idea that erroneous prosaccades are the result of working memory deficits can also account for these findings.

The results of the present study as well as those of Godijn and Kramer (2006) are hard to explain from a reflexive-prosaccade perspective. That is, why are there more erroneous prosaccades to static color singleton targets than to onset or offset targets? Why do participants execute erroneous prosaccades to static targets when there 
are onsets or offsets at the nontarget locations? These can hardly be considered reflexive prosaccades. Furthermore, in Godijn and Kramer (2006) why did participants only execute a substantial proportion of erroneous saccades to onsets or color singletons when they were task-relevant? We have proposed that these errors are best explained as consequences of working memory failure (e.g., Kane et al., 2001; Roberts et al., 1994; Unsworth et al., 2004; Walker et al., 1998). Furthermore, the idea that prosaccades are programmed reflexively in the antisaccade task until a cancellation or inhibition signal is given does not appear to fit well with neurophysiological findings. Several studies have shown that cells in the superior colliculus (SC), a brain region of critical importance for saccade programming, can show both stimulus-related activity and saccade-related activity when monkeys perform an antisaccade task (e.g., Everling, Dorris, Klein \& Munoz, 1999) or a prosaccade task (e.g., Everling et al., 1999; Sparks, Rohrer, \& Zang, 2000). When the prosaccade stimulus is presented stimulus-related activity is automatically elicited. However, before the saccade-related activation starts, the stimulusrelated activity already begins to decay. On trials without erroneous prosaccades the activity of the cells with the prosaccade target in their receptive field continues to decay after the initial stimulus-related response and there is no substantial saccade-related activity (e.g., Everling et al., 1999). The view of a reflexive saccade that runs until an inhibition or cancellation signal is given does not appear to be consistent with these neural cell patterns.

Furthermore, recent work by Schall and colleagues suggests that under certain conditions no saccade is programmed to the prosaccade location. In one study, Schall (2004) trained monkeys to search for a color singleton target and based on the target's orientation to execute a prosaccade or antisaccade. On the basis of single cell recordings, Schall distinguished between two types of neurons in the frontal eye fields (FEF), another region involved in oculomotor control. One group of neurons initially selected the color singleton regardless of the task. On antisaccade trials the activation patterns of these neurons demonstrated a transition from the color singleton to the antisaccade location. A second group of neurons selected the color singleton on prosaccade trials and the antisaccade location on antisaccade trials. These results suggest that one group of neurons is involved in attentional allocation, while another group of neurons is involved in saccade target selection. In another study Juan, Jacobi, and Schall (2004) used the same color singleton search task and evoked saccades by intracortical microstimulation of the monkey FEF. It was found that saccades that were evoked by microstimulation on antisaccade trials deviated to the antisaccade location, but never to the location of the color singleton target. These results provide evidence that erroneous prosaccades are not necessarily programmed on antisaccade trials.

\section{Prosaccade Inhibition}

The view that reflexive prosaccades are automatically programmed in the antisaccade task is typically accompanied by the view that these reflexive prosaccades need to be inhibited in order to generate an antisaccade. Interestingly, neurophysiological studies have provided evidence for inhibition in brain regions involved in oculomotor programming such as the SC (e.g., Munoz \& Fecteau, 2002; Munoz \& Istvan, 1998; Munoz \& Wurtz, 1993). However, this inhibition represents lateral inhibition from neurons that are activated onto other neurons. Specifically, when fixation neurons in the SC are strongly activated, lateral inhibition results in a reduction of activity of saccade-related neurons (e.g., Munoz \& Wurtz, 1993). Thus, oculomotor inhibition may be achieved by activation of fixation cells. Note, however, that this lateral inhibition is not inhibition of any specific saccade program. Instead, it is inhibition of all oculomotor activity. Furthermore, Everling et al. (1999) showed that the activity of fixation-related cells was enhanced prior to the appearance of the target on antisaccade trials relative to prosaccade trials. This finding may help explain why the gap effect is greater for prosaccades than for antisaccades (e.g., Forbes \& Klein, 1996; Reuter-Lorenz, Hughes, \& Fenrich, 1995). The gap effect refers to the finding that, when the fixation stimulus is removed prior to the appearance of the target, saccade latencies are reduced, but more in the prosaccade task than in the antisaccade task. It has been shown that the gap effect is related to a reduction of fixation-related activity (Dorris \& Munoz, 1995; Dorris, Paré, \& Munoz, 1997). Forbes and Klein (1996) suggested that the gap effect may be smaller in the antisaccade task, because the activity of the fixation system is endogenously enhanced.

The difference between prosaccade and antisaccade trials in neural activity prior to the appearance of the target indicates that pre-target preparation plays an important role in antisaccade generation. It is plausible that this is also related to the working memory demands of the antisaccade task. Indeed, the importance of preparing for the antisaccade task prior to the appearance of the target has been well established in several recent fMRI studies, which have revealed differences in neural activation prior to the appearance of the target between the prosaccade task and the antisaccade task (e.g., Curtis \& D'Esposito, 2003; Connolly, Goodale, Menon, \& Munoz, 2002; Desouza, Menon, \& Everling, 2003; Ford, Goltz, Brown, \& Everling, 2005). For example, Ford et al. showed that neural activation was greater on correct antisaccade trials than correct prosaccade trials prior to appearance of the target in several frontal and parietal areas. Furthermore, the preparatory activation of the frontal areas was also greater on correct antisaccade trials than on erroneous prosaccade trials in the antisaccade task.

Taken together, these findings suggest that oculomotor inhibition is involved in the antisaccade task. However, it appears that this inhibition is a general inhibition of oculomotor activity, not of any specific saccade program, which is set-up well before the target is presented. This is contrary to the traditional view of a saccade program that runs until an inhibitory or cancellation signal is given. Nevertheless, these findings do point to the possibility that part of the antisaccade cost may be caused by a difference in the degree to which the fixation system is activated in the prosaccade task relative to the antisaccade 
task. Still, it is clear that this cannot explain the differences in antisaccade costs between the different types of targets in the present study, particularly because the different target conditions were mixed within blocks in Experiments 2-4.

\section{Parallel Versus Serial Processing}

The results of the present study have been interpreted as evidence for a serial allocation of attention from the prosaccade location to the antisaccade location. Although this is primarily a serial model, it is not a model of serial saccade programming. That is, since we do not assume that a prosaccade is programmed on correct antisaccade trials we only assume a single saccade program. In contrast, Massen (2004) has proposed a model of parallel programming of a prosaccade and an antisaccade. Her model predicts that as the antisaccade cost increases so does the proportion of erroneous prosaccades. Massen's model assumes that if prosaccades are slowed down the proportion of erroneous prosaccades and the antisaccade cost are reduced, while if antisaccades are slowed down the proportion of erroneous prosaccades and the antisaccade cost are increased. This is consistent with the present results. However, a closer look at the data reveals that such a parallel model cannot account for the differences we find between the various target conditions. We find a greater antisaccade cost with static targets than with dynamic targets. It would be expected that static targets, relative to dynamic targets, slow down prosaccades more than antisaccades. Massen's parallel model cannot account for the opposite finding in the present study. Although we agree that erroneous prosaccades and the antisaccade cost are related, our explanation for this relation is that they are both caused by interference of working memory processes, that are needed to guide attention to the antisaccade location.

\section{AUTHOR NOTE}

This research was supported by a postdoctoral fellowship from the Arnold and Mabel Beckman Foundation to R.G. We thank Jay Pratt, Richard Abrams, and an anonymous reviewer for helpful comments on an earlier draft of the manuscript. Correspondence concerning this article should be addressed to R. Godijn, Beckman Institute, University of Illinois, 405 N. Mathews Avenue, Urbana, IL 61801 (e-mail: godijn@uiuc.edu).

\section{REFERENCES}

AsaAd, W. T., Rainer, G., \& Miller, E. K. (1998). Neural activity in the primate prefrontal cortex during associative learning. Neuron, 21, 1399-1407.

Connolly, J. D., Goodale, M. A., Menon, R. S., \& Munoz, D. P. (2002). Human fMRI evidence for the neural correlates of preparatory set. Nature Neuroscience, 5, 1254-1255.

Curtis, C. E., \& D'Esposito, M. (2003). Success and failure suppressing reflexive behavior. Journal of Cognitive Neuroscience, 15, 409-418.

Desouza, J. F., Menon, R. S., \& Everling, S. (2003). Preparatory set associated with pro-saccades and anti-saccades in humans investigated with event-related fMRI. Journal of Neurophysiology, 89, 1016-1023.

Deubel, H., \& SchneIder, W. X. (1996). Saccade target selection and object recognition: Evidence for a common attentional mechanism. Vision Research, 36, 1827-1837.

Dorris, M. C., \& Munoz, D. P. (1995). A neural correlate for the gap effect on saccadic reaction times in monkey. Journal of Neurophysiology, 73, 2558-2562.
Dorris, M. C., Paré, M., \& Munoz, D. P. (1997). Neural activity in monkey superior colliculus related to the initiation of saccadic eye movements. Journal of Neuroscience, 17, 8566-8579.

DunCAN, J. (1995). Attention, Intelligence and the frontal lobes. In M.S. Gazzaniga (Ed.) The cognitive neurosciences (pp. 721-733). Cambridge: MIT Press.

ENGLE, R. W. (2000). What is working memory capacity? In H. L. Roediger, J. S. Nairne, I. Neath, \& A. M. Suprenant (Eds.), The nature of remembering: Essays in honor of Robert G. Crowder (pp. 297314). Washington, DC: American Psychological Association.

Engle, R. W., Kane, M. J., \& Tuholski, S. W. (1999). Individual differences in working memory capacity and what they tell us about controlled attention, general fluid intelligence and functions of the prefrontal cortex. In A. Miyake \& P. Shah (Eds.), Models of working memory: Mechanisms of active maintenance and executive control (pp. 102-134). New York: Cambridge University Press.

Everling, S., \& Dorris, M. C., Klein, R. M., \& Munoz, D. P. (1999). Role of primate superior colliculus in preparation and execution of anti-saccades and pro-saccades. Journal of Neuroscience, 19, 2740-2754.

EverLing, S., \& Fischer, B. (1998). The antisaccade: A review of basic research and clinical studies. Neuropsychologia, 36, 885-899.

Everling, S., Spantekow, A., Krappmann, P., \& Flohr, H. (1998) Event-related potentials associated with correct and incorrect responses in a cued antisaccade task. Experimental Brain Research, 118, 27-34.

Fischer, B., \& WeBer, H. (1992). Characteristics of "anti" saccades in man. Experimental Brain Research, 89, 415-424.

Fischer, B., \& WeBer, H. (1996). Effects of precues on error rate and reaction times of antisaccades in human subjects. Experimental Brain Research, 109, 507-512.

Forbes, K., \& KleIn, R. M. (1996). The magnitude of the fixation offset effect with endogenously and exogenously controlled saccades. Journal of Cognitive Neuroscience, 8, 344-352.

Ford, K. A., Goltz, H. C., Brown, M. R., \& EverLing, S. (2005). Neural processes associated with antisaccade task performance investigated with event-related fMRI. Journal of Neurophysiology, 94, 429-440.

GodiJn, R., \& Kramer, A. F. (2006). Prosaccades and antisaccades to onsets and color singletons: evidence that erroneous prosaccades are not reflexive. Experimental Brain Research, 172, 439-448.

Godisn, R., \& Pratt, J. (2002). Endogenous saccades are preceded by shifts of visual attention: evidence from cross-saccadic priming effects. Acta Psychologica, 110, 83-102.

Godis, R., \& Theeuwes, J. (2002). Programming of endogenous and exogenous saccades: evidence for a competitive integration model. Journal of Experimental Psychology: Human Perception \& Performance, 28, 1039-1054.

Goldberg, M. E., Bisley, J., Powell, K. D., Gottlieb, J., \& KusonoKI, M. (2002). The role of the lateral intraparietal area of the monkey in the generation of saccades and visuospatial attention. $A n-$ nals of the New York Academy of Sciences, 956, 205-215.

Goldman-RaKic, P. (1987). Circuitry of primate prefrontal cortex and regulation of behavior by representational memory. In F. Plum (Ed.), Handbook of physiology: The nervous system (pp. 373-417). Bethesda, MD: American Physiological Society.

Guitton, D., Buchtel, H. A., \& Douglas, R. M. (1985). Frontal lobe lesions in man cause difficulties in suppressing reflexive glances and in generating goal directed saccades. Experimental Brain Research, $\mathbf{5 8}, 455-472$.

Hallett, P. E. (1978). Primary and secondary saccades to goals defined by instructions. Vision Research, 18, 1279-1296.

Hallett, P. E., \& Adams, W. D. (1980). The predictability of saccade latency in a novel oculomotor task. Vision Research, 20, 329-339.

Hoffman, J. E., \& Subramaniam, B. (1995). The role of visual attention in saccadic eye movements. Perception \& Psychophysics, 57, 787-795.

Juan, C. H., Shorter-Jacobi, S. M., \& Schall, J. D. (2004). Dissociation of spatial attention and saccade preparation. Proceedings of the National Academy of Sciences, 101, 15541-15544.

Kane, M. J., Bleckley, M. K., Conway, A. R. A., \& Engle, R. W. (2001). A controlled-attention view of working memory capacity. Journal of Experimental Psychology: General, 130, 169-183.

KANE, M. J., \& ENGLE, R. W. (2003). Working-memory capacity and the 
control of attention: The contributions of goal neglect, response competition, and task-set to Stroop interference. Journal of Experimental Psychology: General, 132, 47-70.

Kane, M. J., Poole, B. J., Tuholski, S. W., \& Engle, R. W. (2006). Working memory capacity and the top-down control of visual search: Exploring the boundaries of "executive attention." Journal of Experimental Psychology: Learning, Memory, \& Cognition, 32, 749-777.

Kowler, E., Anderson, E., Dosher, B., \& Blaser, E. (1995). The role of attention in the programming of saccades. Vision Research, 35, 1897-1916.

Kramer, A. F., Gonzalez de Sather, J. C. M., \& Cassavaugh, N. D. (2005). Development of Attentional and Oculomotor Control. Developmental Psychology, 41, 760-762.

Kramer, A. F., Hahn, S., Irwin, D. E., \& Theeuwes, J. (1999). Attentional capture and aging: Implications for visual search performance and oculomotor control. Psychology \& Aging, 14, 135-154.

LynCh, J. C., Mountcastle, V. B., Talbot, W. H., \& Yin, T. C. (1977). Parietal lobe mechanisms for directed visual attention. Journal of Neurophysiology, 40, 362-389.

MASSEN, C. (2004). Parallel programming of exogenous and endogenous components in the antisaccade task. Quarterly Journal of Experimental Psychology, 57A, 475-498.

Milea, D., Lehericy, S., Rivaud-Pechoux, S., Duffau, H., Lobel, E., Capelle, L., Marsault, C., Berthoz, A., \& PierrotDeseilligny, C. (2003). Antisaccade deficit after anterior cingulate cortex resection. NeuroReport, 14, 283-287.

MoKLeR, A., \& Fischer, B. (1999). The recognition and correction of involuntary prosaccades in an anti-saccade task. Experimental Brain Research, 125, 511-516.

Munoz, D. P., Broughton, J. R., Goldring, J. E., \& Armstrong, I. T. (1998). Age-related performance of human subjects on saccadic eye movement tasks. Experimental Brain Research, 121, 391-400.

MunOz, D. P., \& FeCTEAU, J. H. (2002). Vying for dominance: dynamic interactions control visual fixation and saccadic initiation in the superior colliculus. Progress in Brain Research, 140, 3-19.

Munoz, D. P., \& IstVan, P. J. (1998). Lateral inhibitory interactions in the intermediate layers of the monkey superior colliculus. Journal of Comparative Neurology, 276, 169-187.

Munoz, D. P., \& Wurtz, R. H. (1993). Fixation cells in monkey superior colliculus. II. Reversible activation and deactivation. Journal of Neurophysiology, 70, 576-589.

OlK, B., \& KingSTONE, A. (2003). Why are antisaccades slower than prosaccades? A novel finding using a new paradigm. NeuroReport, 14, 151-155.

Pierrot-Deseilligny, C., Ploner, C. J., Muri, R. M., Gaymard, B., \& Rivaud-Pechoux, S. (2002). Effects of cortical lesions on saccadic eye movements in humans. Annals of the New York Academy of Sciences, 956, 216-229.

Pierrot-Deseilligny, C., Rivaud, S., Gaymard, B., \& Agid, Y. (1991). Cortical control of reflexive visually-guided saccades. Brain, 114, 1473-1485.
Posner, M. I. (1980). Orienting of attention. Quarterly Journal of Experimental Psychology, 32, 3-25.

Pratt, J., \& Hirshhorn, M. (2003). Examining the time course of facilitation and inhibition with simultaneous onset and offset cues. Psychological Research, 67, 261-265.

Pratt, J., \& Trottier, L. (2005). Pro-saccades and anti-saccades to onset and offset targets. Vision Research, 45, 765-774.

RaO, S. C., Rainer, G., \& Miller, E. K. (1997). Integration of what and where in the primate prefrontal cortex. Science, 276, 821-824.

Reuter-Lorenz, Hughes, H. C, \& Fenrich, R. (1995). The reduction of saccadic latency by prior offset of the fixation point: an analysis of the gap effect. Perception \& Psychophysics, 49, 167-175.

Roberts, R. J., Hager, L. C., \& Heron, C. (1994). Prefrontal cognitive processes: Working memory and inhibition in the antisaccade task. Journal of Experimental Psychology: General, 123, 374-393.

SAmuel, A. G., \& WeINER, S. K. (2001). Attentional consequences of object appearance and disappearance. Journal of Experimental Psychology: Human Perception \& Performance, 27, 1433-1451.

SCHALL, J. D. (2004). On the role of frontal eye field in guiding attention and saccades. Vision Research, 44, 1453-1467.

Sparks, D., Rohrer, W. H., \& Zhang, Y. (2000). The role of the superior colliculus in saccade initiation: a study of express saccades and the gap effect. Vision Research, 40, 2763-2777.

THeEUwES, J. (1995). Abrupt luminance change pops out; abrupt color change does not. Perception \& Pyschophysics, 57, 637-644.

Theeuwes, J., Kramer, A. F., Hahn, S., \& Irwin, D. E. (1998). Our eyes do not always go where we want them to go: capture of the eyes by new objects. Psychological Science, 9, 379-385.

Unsworth, N., Schrock, C. S., \& Engle, R. W. (2004). Working memory capacity and the antisaccade task: individual differences in voluntary saccade control. Journal of Experimental Psychology: Learning, Memory, \& Cognition, 30, 1302-1321.

Walker, R., Husain, M., Hodgson, T. L., Harrison, J., \& Kennard, C. (1998). Saccadic eye movement and working memory deficits following damage to human prefrontal cortex. Neuropsychologia, 36, 1141-1159.

Walker, R., Walker, D., Husain. M, \& Kennard, C. (2000) Control of voluntary and reflexive saccades. Experimental Brain Research, 130, 540-544.

Yantis, S. (1996). Attentional capture in vision. In A. F. Kramer, M. G. H. Coles, \& G. Logan (Eds.), Converging operations in the study of visual selective attention (pp. 45-76). Washington, DC: American Psychological Association.

Yantis, S., Schwarzbach, J., Serences, J. T., Carlson, R. L., Steinmetz, M. A., Pekar, J. J., \& Courtney, S. M. (2002). Transient neural activity in human parietal cortex during spatial attention shifts. Nature Neuroscience, 5, 995-1002.

(Manuscript received January 12, 2006; revision accepted for publication December 12, 2006.) 